\title{
Implicaciones de Género en la Sociedad de la Información: Un Análisis desde los Determinantes de Uso de Internet en Chile y México
}

\author{
Martha Sánchez Galvis'
}

\begin{abstract}
This document presents the gender differences in Internet use for some Latin American countries and, through an empirical exercise, validates these differences, as well as the gender interaction with the determinants of Internet use. For this purpose, a model of interaction effects is applied to Mexico and Chile, with the income as one of the key determinants in the use of Internet. The main findings suggest that the positive effect of the income on the probability of use of Internet is greater for men than for women. This is one of the first papers that show the status of gender indicators and Internet use in the region. Additionally, this is the first empirical validation of gender gaps given by using models based on information from ICT households' surveys of Mexico and Chile. Thus, based on findings on the gender implications in Internet use, we raised the discussion to public policy guidelines for the region.
\end{abstract}

Keywords: gender; digital divide; internet diffusion; interaction terms; Latin America.

\section{Resumen}

Este documento presenta las diferencias de género en el uso de Internet para algunos países de América Latina y mediante un ejercicio empírico valida estas diferencias, así como la interacción del género con los determinantes de uso de Internet. Para esto se aplica a los casos de México y Chile un modelo de Interaction effects, con el ingreso como uno de los principales determinantes de uso de Internet. Los principales hallazgos sugieren que el efecto positivo que tiene el ingreso sobre la probabilidad de uso de Internet, resulta mayor para los hombres que para las mujeres. Este es uno de los primeros documentos en mostrar el estado de los indicadores de género y uso de Internet en la región. Adicionalmente es el primero en validar las diferencias de género empíricamente a través de modelos que utilizan la información de TIC de las encuestas de hogares de México y Chile. De esta manera, con base en los hallazgos sobre las implicaciones de género en el uso de Internet se hacen algunas reflexiones con orientación de políticas públicas para la región.

Palabras clave: género; brecha digital; difusión de internet; términos de interacción; América Latina.

\footnotetext{
I Consultora de la Comisión Económica para América Latina y el Caribe de las Naciones Unidas CEPAL, División de Desarrollo Productivo y Empresarial. Programa Sociedad de la Información. Santiago de Chile. Tel: +56 9 778I 0222. Email: mjsanchezg@gmail.com
} 


\section{Introducción}

La perspectiva de género es un tema emergente que desde algunas décadas atrás se ha consolidado como una corriente transversal a las diferentes problemáticas de la sociedad. Poco a poco se le ha venido reconociendo a las mujeres un rol igualitario en la sociedad, rol que ha sido vulnerado históricamente en ámbitos que abarcan desde los derechos a la salud, la educación, a la vida libre de violencias, hasta su participación política y económica, y hoy en día, hasta sus derechos en la Sociedad de la Información.

Para lograr la restitución de estos derechos de igualdad, los gobiernos cuentan con algunas herramientas de política pública. En particular, en materia de equidad de género los países de América Latina tienen como referencia de sus políticas públicas responder con algunos lineamientos y tratados internacionales, como son los Objetivos de Desarrollo del Milenio y los compromisos de Beijing. Dentro de éstos se encuentran los acuerdos que propenden por aumentar el acceso de la mujer a las tecnologías de información y comunicación (TIC), y a aumentar su goce de los beneficios que deriva la sociedad de la información.

En este sentido, la difusión de las TIC, ha venido cobrando fuerza como objeto de estudio para el diseño de políticas para investigadores y académicos, entre otros actores de la sociedad, principalmente referidas al acceso y al uso de las TIC, tanto por hogares, como por individuos.

En materia de acceso a las TIC se ha podido identificar que para algunos países la brecha digital, entre mujeres y hombres ha venido desapareciendo desde finales de la década pasada (Ono y Zavodny, 2003; Bimber, 2000). No obstante, para América Latina los estudios se han concentrado en abordar el análisis de la brecha digital de una manera uniforme, sin profundizar en las diferencias de género que pueden estar matizando dicha brecha. Por lo tanto, y a raíz de los avances de la región en el acceso de los hogares $y$ las personas a las TIC, surgen nuevos desafíos para los países en materia de análisis del uso diferencial de las TIC y sus implicaciones para el desarrollo.

En este sentido, se ha discutido ampliamente el hecho de que las TIC son generadoras de nuevas formas de desigualdad entre mujeres y hombres, reflejadas en las diferencias en oportunidades de acceso y en el uso diferencial de estas tecnologías, específicamente en el uso diferencial de Internet (Hargittai y Shafer, 2006; Castaño, 2005). Esto ha sido mayormente estudiado en los países desarrollados, sin embargo, para América Latina, hasta ahora no se tiene un análisis profundo de esta problemática.

De esta manera, resulta imperioso un análisis del acceso y el uso diferencial a las TIC para mujeres y hombres en la región, ya que estas tecnologías se reconocen como potenciales herramientas de desarrollo, superación de la pobreza e inclusión social que, dependiendo de las políticas de acceso y los patrones de uso, podrían incidir positiva o negativamente en las brechas de género ya existentes en América Latina.

La literatura que se ha concentrado en analizar los patrones de difusión de las TIC en América Latina, ha buscado identificar los determinantes de acceso y uso, principalmente a tecnologías como computadora e Internet, de esta manera se ha podido construir un perfil para cierto número de países de la región que cuentan con la información necesaria para llevar a cabo los análisis de demanda de servicios TIC.

No obstante, estos perfiles no han podido identificar si existe un componente de género que esté matizando los determinantes de uso de las tecnologías, es decir, si existe un efecto de género que haga que los principales determinantes de uso de TIC muestren comportamientos diferentes para mujeres y hombres. Este documento presenta evidencia del efecto de género en los patrones de uso de Internet para Chile y México y cuantifica la magnitud del efecto con relación a uno de los principales determinantes de uso de Internet, el ingreso.

De esta manera, además de esta introducción, este documento presenta una segunda sección sobre el estado del arte de los estudios relativos a género y TIC, que recoge los principales hallazgos $y$ las tendencias identificadas, en el uso y acceso a las TIC, para mujeres y hombres. La tercera sección da un panorama regional de la información disponible para el análisis y de los principales indicadores de uso de Internet. La cuarta sección presenta el ejercicio econométrico que fue utilizado para medir los efectos de género en el ingreso como principal 
determinante de uso de Internet en Chile y México. Posteriormente se presentan los resultados y algunas conclusiones y consideraciones finales.

\section{Estado del Arte}

Las TIC, y en particular el uso de Internet, conllevan beneficios relativos al conocimiento y acceso a servicios gubernamentales, acceso al mercado de trabajo, información y programas de salud, oportunidades de educación, formación de capital humano, programas y oportunidades de generación de ingresos, información sobre democracia y participación política, acceso a políticas y programas sociales, y entretenimiento, entre otros. Estos beneficios son potenciales herramientas de desarrollo que pueden disminuir o aumentar las brechas ya existentes.

En materia de género el acceso diferenciado a estas herramientas tendría dos matices: el primero referido a los determinantes del uso diferenciado, es decir los factores que identifican esta diferencia (demográficos, sociales, económicos, culturales, etc.); el segundo, referido a las implicaciones que éste tenga sobre la brecha de género y sobre los procesos de desarrollo de mujeres y hombres en la sociedad.

Al respecto, la literatura internacional ha registrado diversas implicaciones de género que caracterizan la sociedad de la información. Algunos estudios han logrado identificar las diferencias de género desde el proceso mismo del auto reconocimiento frente a las tecnologías, en cuanto a habilidades y confianza, hasta la eficacia en el uso de las mismas (Shashaani, 1997; Kennedy, Wellman y Klement, 2003).

Los primeros hallazgos sugieren que en promedio, las mujeres sienten menos confianza y menos habilidades frente al uso de Internet como medio de consecución de información para el desarrollo, con respecto a los hombres, y en efecto, en algunos casos la eficacia de los hombres en el uso de Internet resultó mayor que la de las mujeres (Hargittai y Shafer, 2006).

Sin embargo, las diferencias de género no solamente dependen de las habilidades de hombres y mujeres para usar las herramientas. De hecho, en América Latina la problemática de género se debe en gran parte a la desigualdad de oportunidades en diferentes ámbitos del desarrollo (e.g., participación política, trabajo e ingresos), desigualdades que llevan detrás una construcción ancestral de roles para los géneros y que no están relacionadas con las capacidades individuales de las mujeres.

En el caso de las TIC, las oportunidades están fuertemente ligadas a la capacidad de los hogares de acceder a las TIC como un conjunto, como un todo. Sin embargo, hay evidencia de que, no obstante haya acceso a las TIC en el hogar, las mujeres tendrían en promedio menores oportunidades de uso de dichas tecnologías. (Chen, 2004; OECD, 2007). Esto mismo se pudo comprobar para América Latina (Grazzi, 2009).

Lo anterior puede estar altamente ligado a los roles de género que determinan el uso del tiempo de hombres y mujeres. El tiempo que las mujeres tendrían disponible para uso de Internet en el hogar sería más restringido que el que tendrían los hombres, por cuanto la sociedad ha atribuido el rol del trabajo reproductivo y el de la economía del cuidado a las mujeres. Las encuestas de uso del tiempo han podido identificar que sumando las horas de trabajo remunerado y no remunerado de mujeres $y$ hombres, las mujeres tienen, en promedio, más horas de trabajo y por lo tanto, menos horas disponibles para otras actividades (ocio), entre ellas el uso de Internet.

En el mismo sentido, se han podido identificar patrones de uso que dejan ver las oportunidades, el comportamiento y las preferencias de hombres y mujeres al momento de navegar en Internet.

Ono y Zavodny (2003) mostraron que aunque en Estados Unidos la brecha de género en acceso casi ha desaparecido y la de uso ha disminuido, es evidente que algunas diferencias prevalecen. De hecho, en materia de uso diferencial, Kennedy et al. (2003), encontraron que en Canadá y Estados Unidos, las mujeres utilizan Internet más para fines sociales, de salud y de cuidado de los menores, mientras que los hombres se concentran en actividades de entretenimiento.

Así mismo, la OECD (2007), en su informe al Working Party on the Information Economy, reveló que en los países miembros, las mujeres tienen mayor incidencia en actividades de compras on-line y actividades de salud, mientras que los hombres concentraron el uso de Internet en información sobre deportes y video juegos. En resumen, la mayoría de los estudios muestran que las mujeres son menos uso-intensivas de Internet con

ISSN: 07I 8-2724. (http://www.jotmi.org)

Journal of Technology Management \& Innovation () Universidad Alberto Hurtado, Facultad de Economía y Negocios 
respecto a los hombres y que, aunque la brecha de acceso a las TIC no es evidente en todos los casos, si lo es la brecha de uso.

Para América Latina, estudios recientes demostraron la tendencia de las mujeres a concentrar su uso de Internet en actividades relativas a la educación, mientras que para los hombres las principales actividades online resultaron ser el entretenimiento y la banca electrónica. (Grazzi, 2009 y Sánchez, 2009). Esto se puede generalizar como tendencia de las mujeres a concentrar el uso de Internet en relaciones humanas y sociales, mientras que los hombres concentran su uso de Internet en las actividades relacionadas con la experiencia en sí que éste les brinda.

Estas diferencias pueden eventualmente afectar también las potencialidades del uso de las TIC para el desarrollo, específicamente, su uso como factores de empoderamiento para las mujeres y por lo tanto, para la región resulta pertinente estudiar este fenómeno. Adicionalmente, es preciso indagar por el comportamiento de los principales determinantes de uso de TIC, como el ingreso y el nivel educativo, para ver la influencia de éstos en la difusión de TIC entre mujeres y hombres de la región.

\section{Uso de Internet por Mujeres y Hombres en América Latina}

La información de demanda de TIC por hogares e individuos está disponible principalmente en dos fuentes de información: las encuestas de hogares y las encuestas específicas de TIC de los países. Las encuestas de hogares de los países son investigaciones multipropósito que cubren diferentes temáticas. Generalmente, estas encuestas se centran en la medición de indicadores sociales y económicos (e.g., tasas de ocupación, alfabetismo, pobreza, etc.). Sin embargo, muchas de ellas han incorporado dentro de sus módulos temáticos o temporales, el acceso y el uso de las TIC. Esto ha permitido hacer un seguimiento periódico de las TIC y un análisis complementario con variables socioeconómicas propias de estas encuestas.

Por otro lado, las encuestas específicas de TIC han sido incorporadas en algunos países que han visto la necesidad de hacer una investigación más profunda del acceso y el uso de las TIC tanto para los hogares, como para los individuos. Estas encuestas específicas permiten incorporar preguntas adicionales que dan una caracterización más amplia de los patrones de uso y acceso a las $\mathrm{TIC}^{2}$.

Con esta información se presenta un breve panorama de los indicadores de difusión de Internet para hombres y mujeres de los países de la región. Para esto se tomó el último año disponible de las encuestas de los países que contaban con preguntas de uso de computadora e Internet en sus investigaciones de manera simultánea. Estas encuestas fueron: Chile 2006, México 2007, Panamá 2007, República Dominicana 2005 y Uruguay 2006³. Finalmente, se analizan algunas variables de caracterización sociodemográfica y de patrones de uso de Internet con el fin de profundizar en el análisis de las brechas de acceso y uso de computadora e Internet de los países, para esto se utilizaron las encuestas de hogares de los países que indagan por el uso de Internet y por las variables de caracterización al mismo tiempo. Esta caracterización poblacional se hizo con base en las encuestas de hogares de Brasil 2005, Chile 2006, El Salvador 2006, México 2007 y Panamá 2007.

El gráfico I muestra las diferencias entre mujeres y hombres del uso individual de Internet. Chile es el país en donde tanto hombres como mujeres tienen mayores índices de uso de Internet con niveles entre el $30 \%$ y e $40 \%$, seguido por Uruguay (entre $20 \%$ y $30 \%$ ), México y Panamá (cerca del 20\%) y República Dominicana (entre el $10 \%$ y $20 \%)^{4}$.

\footnotetext{
2 Una comparación detallada de la tipología de encuestas aplicadas en la región, sus ventajas y sus desventajas, se encuentra en el "Compendio de Prácticas sobre Implementación de Preguntas de TIC en Encuestas de Hogares y Empresas" Revisión 2009, documento de OSILAC que se encuentra en versión preliminar en http://www.eclac.cl/socinfo/noticias/paginas/0/35880/CompendioP racticas Mar2009.pdf. (Cristancho y Sánchez, 2009). Todas las encuestas utilizadas en este estudio son de propósitos múltiples excepto la encuesta de México que es una encuesta especializada en TIC que está adjunta a una encuesta de empleo (Ver anexo I)

${ }^{3}$ Nótese que para el caso de Uruguay se utilizó la encuesta de 2006 y no la de 2007 como se hizo para el análisis de acceso, esto debido a que la encuesta uruguaya en 2007 no indagó sobre el uso de computadora mientras que en el 2006 si lo hizo.

${ }^{4}$ Con el fin de favorecer la comparabilidad entre las poblaciones de mujeres y hombres y para anular el efecto de la pirámide poblacional, los indicadores para cada sexo fueron calculados con respecto a su misma población universo.
}

ISSN: 07I 8-2724. (http://www.jotmi.org)

Journal of Technology Management \& Innovation (C) Universidad Alberto Hurtado, Facultad de Economía y Negocios 


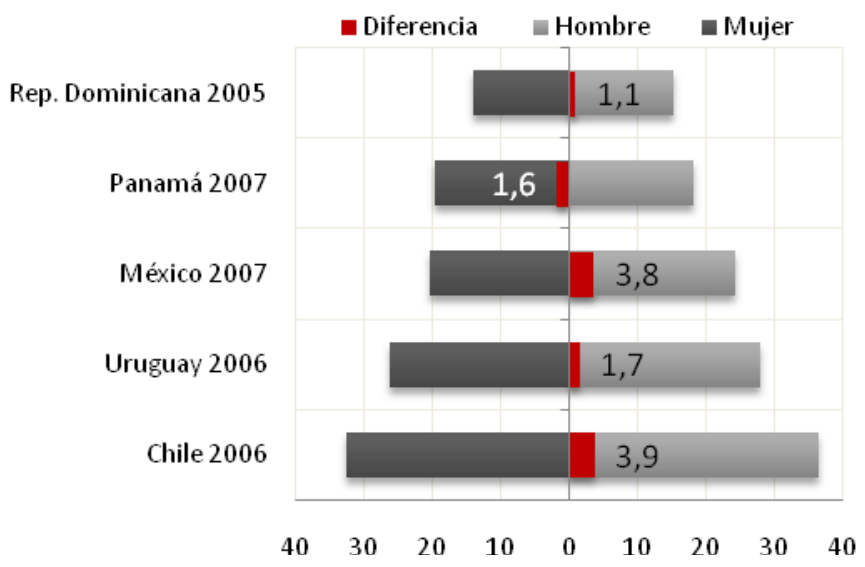

Gráfico I. Porcentaje de mujeres y hombres que usan Internet en América Latina, entre 2005 y 2007 Fuente: Cálculos propios con base en Sistema de Información OSILAC

Ahora bien, con excepción de Panamá, los países en estudio muestran una diferencia en el uso de Internet que favorece a los hombres, independientemente del nivel de uso de Internet del país, de hecho, aquellos países con más alto grado de difusión de Internet (Chile y México), son quienes muestran las mayores diferencias por género. Cabe anotar que las diferencias a favor de los hombres se acentúan cuando se hace el análisis para uso de Computadora. (Sánchez, 2009).

Una aproximación matemática permite una mejor comprensión de lo que significan estas brechas. El diferencial que se presenta a continuación es una herramienta de análisis para cuantificar porcentualmente la brecha entre dos poblaciones, en este caso mujeres y hombres, y se puede expresar como sigue:

$$
\lambda=\left[\left(\frac{i_{m x}}{i_{h x}}\right)-1\right] * 100
$$

En donde, $i_{m x}$ es el indicador de uso de la tecnología $x$ por parte de las mujeres, e $i_{h x}$ es el indicador de uso de la tecnología $\mathrm{x}$ por parte de los hombres. El diferencial $\lambda$ entonces, puede tomar valores positivos o negativos dependiendo de la población que tenga mejores indicadores de uso de la tecnología $x$.

Nótese que de acuerdo a la fórmula del diferencial, ante un mayor indicador de uso de las mujeres el diferencial tomará valores positivos, en caso contrario tomará valores negativos, es decir cuando el diferencial favorezca a los hombres, y será cero ante la presencia de equidad.

El gráfico 2 muestra los resultados del cálculo de los diferenciales de uso de computadora y uso de Internet por sexo. Allí se observa cómo México, Chile, República Dominicana y Uruguay muestran un diferencial negativo para las mujeres en uso de Internet, el cuál varía entre el $6 \%$ y el $16 \%$. En otras palabras, las mujeres en Chile utilizan Internet cerca de un $11 \%$ menos que los hombres. En el caso de México esta diferencia es mayor, las mujeres utilizan casi un $16 \%$ menos Internet que los hombres.

Como se mencionó anteriormente, esta diferencia no está relacionada con la distribución por sexo de la población y por lo tanto lo que refleja es que en promedio las mujeres usan menos el Internet que los hombres siendo este fenómeno más profundo en los casos de México y Chile. Sin embargo, este diferencial no da información sobre las posibles causas de la desigualdad y de sus características. 


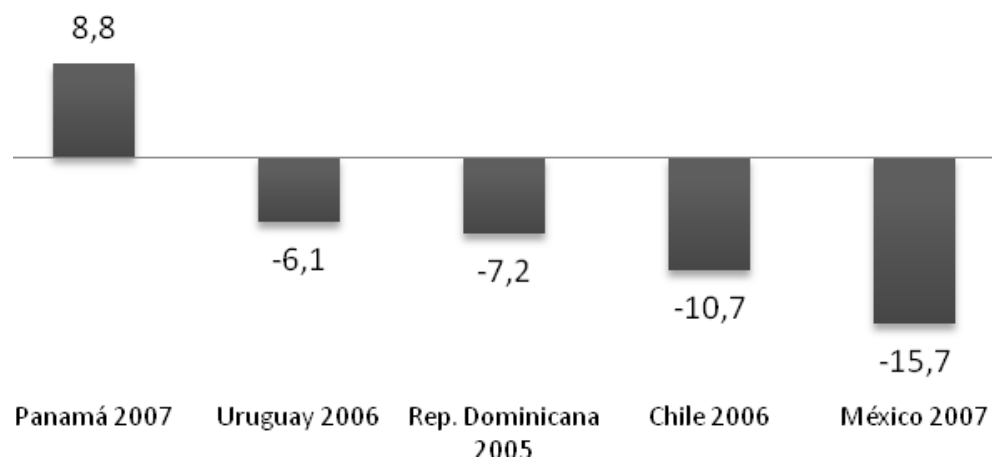

Gráfico 2. Diferencial de género de uso de computadora e Internet para jefas y jefes de hogar en América Latina, entre 2005 y 2007

Fuente: Cálculos propios con base en Sistema de Información OSILAC

Con el fin de profundizar más en estas brechas de uso de Internet por sexo, a continuación se hace la caracterización del uso de Internet de hombres y mujeres, con las variables socio-demográficas más relevantes.

Las brechas de género son diferencias que se reflejan en aspectos de la vida de mujeres y hombres a razón básicamente de su condición biológica, de su sexo, independientemente de otras características sociales, culturales y económicas. La discriminación de género es aquella que se infiere en contra de las mujeres solamente por el hecho de serlo. Por esta razón al hacer los análisis de género se debe también tener cuidado en aislar los efectos de otras variables sobre el indicador que se esté estudiando.
En este sentido, es necesario caracterizar la población femenina y masculina haciendo un análisis de poblaciones con características similares y resaltando las variables que están manifestando diferencias que puedan indicar un patrón de comportamiento, una tendencia o una brecha en el uso de Internet.

El gráfico 3 muestra el uso de Internet para hombres y mujeres según la zona geográfica en la que viven. La primera brecha que se puede identificar es la brecha urbano-rural. Para todos los países en estudio, los indicadores de uso de Internet son mayores en el área urbana, mostrando diferencias que van desde 5 puntos porcentuales (El Salvador), hasta 22 puntos porcentuales (Chile), en promedio.

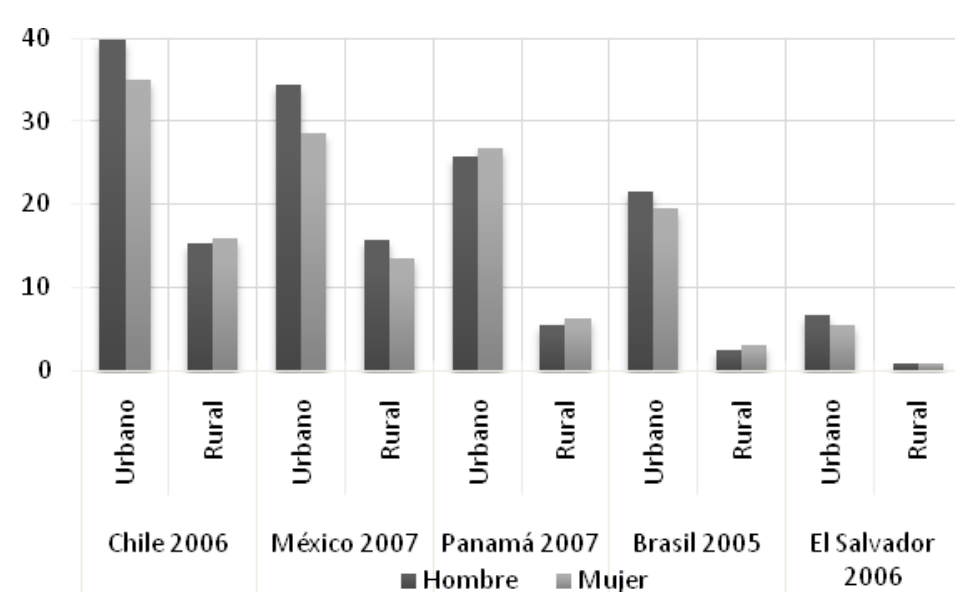

Gráfico 3 Porcentaje de uso de Internet según zona y sexo América Latina, entre 2005 y 2007

Fuente: Cálculos propios con base en Sistema de Información OSILAC 
Con respecto a las diferencias de sexo, con excepción de Panamá, todos los países muestran indicadores de uso de Internet mayores para los hombres en las áreas urbanas, que son precisamente las que tienen altos índices de acceso y uso. Las diferencias porcentuales más marcadas, entre hombres y mujeres, en las zonas urbanas las presentan nuevamente México (5.7 puntos) y Chile (4.9 puntos).

Por otro lado, la brecha de género en las zonas rurales favorece a las mujeres pero en menores magnitudes, excepto para el caso de México que presenta brechas a favor de los hombres también a nivel rural.

Al parecer el hecho de que las zonas rurales tengan todavía niveles tan bajos de penetración en la mayoría de los países de América Latina, muestra una posición más igualitaria de las mujeres respecto a los hombres, sin embargo, esto intuitivamente se puede interpretar como el resultado de un mayor peso relativo de otros factores en las áreas rurales, como la infraestructura, la educación de los individuos y los ingresos de los hogares, frente al peso del factor de género.

Ahora bien, la literatura de TIC en general ha reconocido algunos determinantes del acceso y uso, en particular se han confirmado la edad, el nivel educativo y el ingreso como determinantes de la difusión de las tecnologías en la región (Grazzi y Vergara, 2008). A continuación se exponen los principales resultados de uso de Internet para hombres y mujeres y su comportamiento según estas variables determinantes.

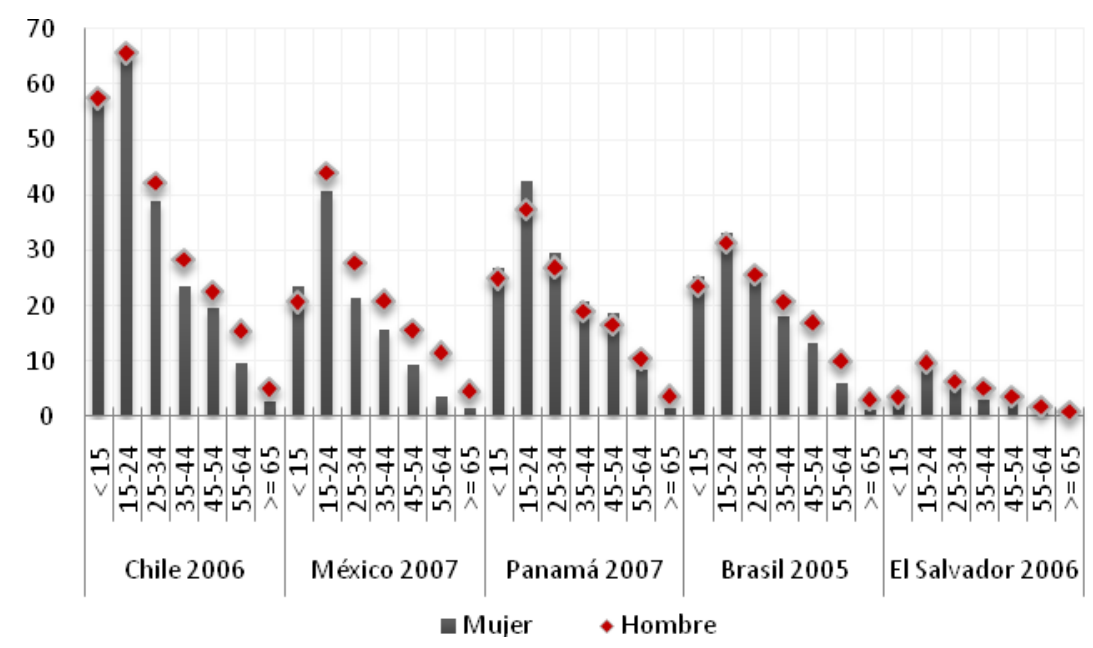

Gráfico 4. Porcentaje de uso de Internet según rangos de edad y sexo en América Latina, entre 2005 y $2007^{7}$.

Fuente: Cálculos propios con base en Sistema de Información OSILAC

El gráfico 4 muestra el porcentaje de uso de Internet para los sexos según grupos de edad decenales, con excepción de los límites inferior y superior que tienen un rango de edad definido 5 .

\footnotetext{
${ }^{5}$ Este rango se amplió aprovechando la disponibilidad de información de los países en estudio, y siguiendo la recomendación del Partnership para la Medición de las TIC para el Desarrollo, a cerca de presentar los indicadores de difusión de TIC con unos rangos mínimos que incluyan a las personas entre los 15 y 74 años: “...En aras de la uniformidad, se propone ese rango mínimo de edad de los individuos. Es posible que muchos países en desarrollo deseen ampliarlo, si poseen recursos suficientes para ello y necesitan datos complementarios."
}

Los datos confirman la existencia de una ${ }^{6}$ brecha generacional, las personas menores de 35 años tienen, en promedio, mayores índices de uso de Internet que el resto de la población en todos los países en estudio. Las y los jóvenes entre 15 y 24 años son quienes muestran los

(Partnership para la medición de las TIC para el desarrollo, 2005, p33).

${ }^{6}$ Para controlar el efecto de la distribución de la población en términos absolutos, los cálculos de los indicadores se hicieron separadamente para hombres y mujeres y se trabajó a nivel de participaciones porcentuales para cada categoría de análisis. Esto se hizo para todos los cálculos de caracterización poblacional. 
índices más altos, alcanzando tasas de uso de Internet de más del $65 \%$, como en el caso de Chile.

Como se puede observar en el gráfico 4, los hombres tienen, en la mayoría de los casos, tasas de uso de Internet mayores a las de sus pares mujeres. Esto es así para todos los rangos de edad de Chile. En México solamente los menores de 15 años tienen un menor porcentaje de uso de Internet que su contraparte femenina, y en Brasil ocurre lo mismo pero para los dos primeros grupos de edad.

Por el contrario, Panamá confirma su tendencia de brecha favorable para las mujeres, mostrando índices superiores para ellas en todos los rangos de edad, con excepción de las mayores de 55 años. El Salvador por su parte muestra leves diferencias, tanto entre los grupos de edad, como entre los sexos.
Las diferencias más significativas en el uso de Internet por edades a favor de los hombres están en México, superando los 6 puntos porcentuales para personas entre los 25 y los 64 años, mientras que la brecha más favorable para las mujeres la tienen las jóvenes de Panamá entre los 15 y los 24 años (4.9 puntos).

De otro lado, el nivel educativo es otro de los factores que ha sido identificado como determinante del uso de Internet, en particular la literatura sugiere que a mayor nivel educativo, mayor es la probabilidad de usar Internet. En efecto, se observa una amplia brecha entre quienes tienen algún grado de educación y quienes no lo tienen. De hecho, el mayor índice de uso de Internet lo presentan las personas con educación superior, sobrepasando de manera importante los indicadores de quienes tienen educación primaria o secundaria. (Gráfico 5).

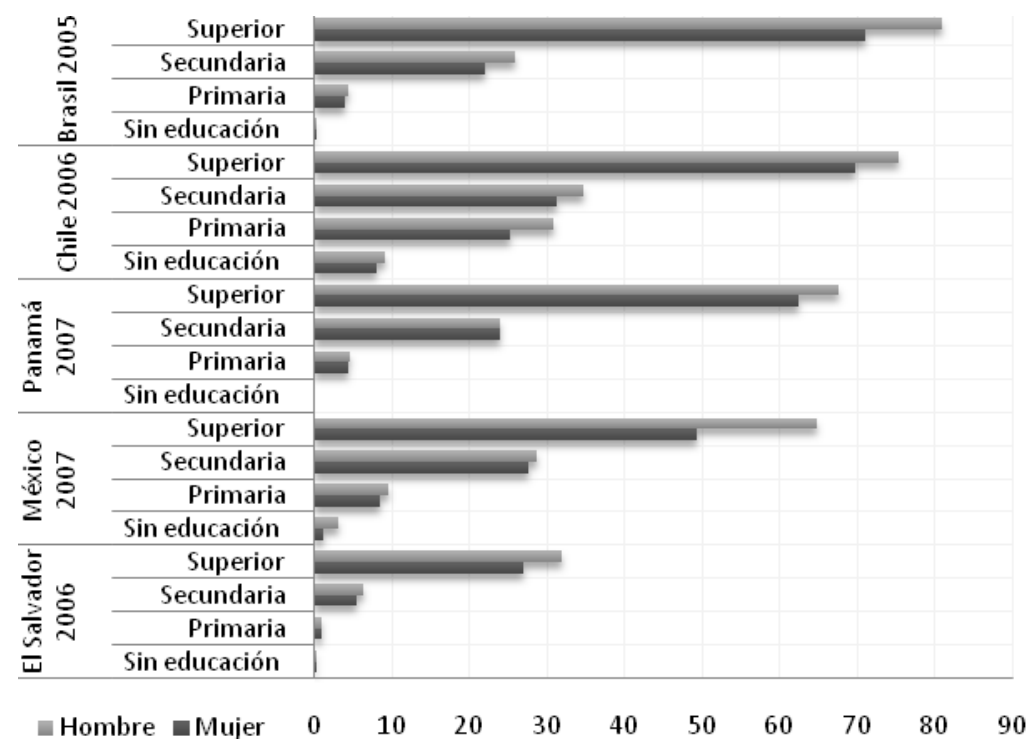

Gráfico 5. Porcentaje de uso de Internet según nivel educativo y sexo en América Latina, entre 2005 y 2007 Fuente: Cálculos propios con base en Sistema de Información OSILAC

Para el caso de Brasil, la diferencia de tasas de uso de Internet entre personas con educación superior $y$ secundaria es de 52 puntos porcentuales (en promedio), seguido por Panamá y Chile con una diferencia de cerca de 40 puntos, y finalmente México y el Salvador con diferencias que oscilan entre 28 y 23 puntos porcentuales, respectivamente. Esta brecha educacional es una de las mayores en términos de difusión de Internet entre grupos poblacionales, prácticamente quienes no tienen por lo menos educación secundaria, no tienen altas posibilidades de ser usuarios frecuentes de Internet ${ }^{7}$.

De la misma manera, las diferencias de género también son evidentes. Para todos los países y para todos los niveles educativos las tasas de uso de Internet son mayores en los

\footnotetext{
${ }^{7}$ Con excepción del caso de Chile que tiene relativamente altos niveles de difusión de Internet incluso para su población con educación primaria.
} 
hombres que en las mujeres. De estas diferencias las mayores las presentan hombres y mujeres con educación superior en México y Brasil con brechas favorables para los hombres de 16 y 10 puntos porcentuales, respectivamente. En otras palabras, en términos de diferencial, las mujeres con educación superior en México y Brasil, utilizan Internet un $24 \%$ y un $12 \%$ menos que sus pares masculinos, respectivamente.

Adicionalmente, es importante señalar que en algunos casos se encuentra paridad entre las tasas. Este es el caso de mujeres y hombres sin educación, y con educación primaria en Panamá, El Salvador y Brasil, quienes tienen equitativamente bajos índices de uso de Internet. En este punto puede estar apareciendo el mismo efecto que se observó en la brecha urbano-rural, en donde otras variables tienen un mayor peso que el del sexo de la persona, en este caso, probablemente, el nivel de ingreso.

Al ver los indicadores según quintiles de ingreso, se confirma que a mayor nivel de ingreso, mayor es el uso de Internet, en correspondencia con la literatura. (Gráfico 6). La población en el quintil más alto de ingresos $(V)$ es la que presenta los mayores índices de uso de Internet para todos los países. En promedio, el quintil $V$ muestra diferencias de 39,34 y 32 puntos porcentuales con respecto al resto de la población, esto para los casos de Brasil, Panamá y México, respectivamente.

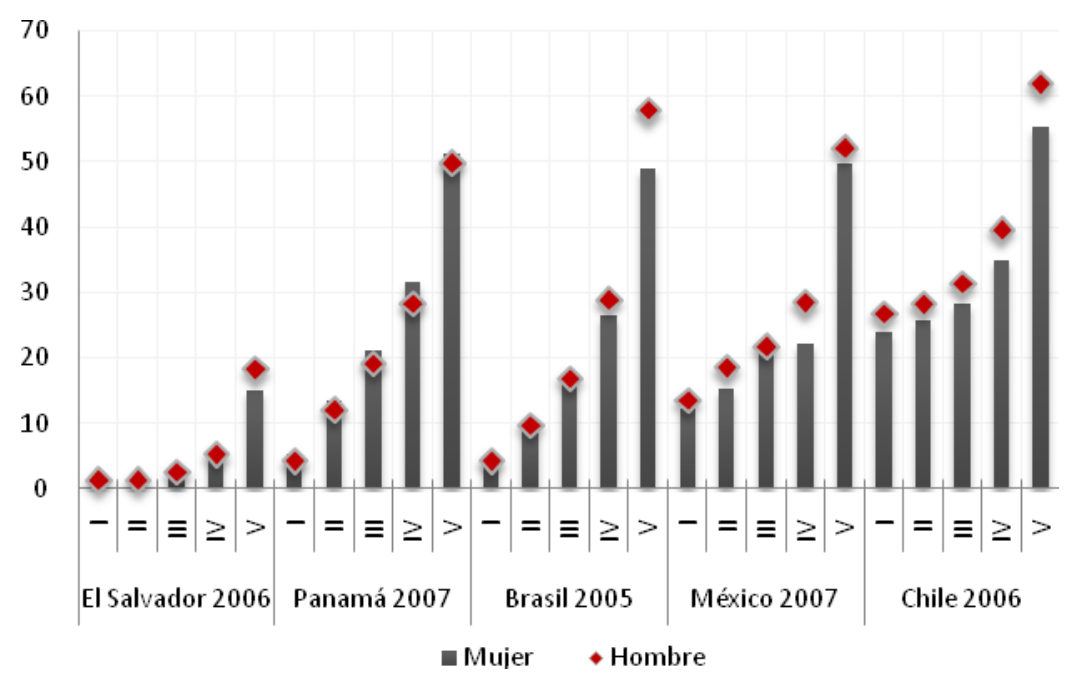

Gráfico 6. Porcentaje de uso de Internet según quintiles de ingreso y sexo en América Latina, entre 2005 y $2007^{9}$

Fuente: Cálculos propios con base en Sistema de Información OSILAC

Por género, las diferencias en el uso de Internet a favor ${ }^{8}$ de los hombres, en cada quintil de ingreso, nuevamente sobresalen en Chile, México y Brasil. En donde, independientemente del nivel de ingreso, las mujeres tienen menores tasas de uso de esta tecnología. Las mayores diferencias entre mujeres $y$ hombres se presentan en el cuarto y quinto quintil, oscilando entre los 5 y los 9 puntos porcentuales.

Por otro lado, Panamá exhibe de nuevo una brecha positiva para las mujeres, aunque en menores

\footnotetext{
${ }^{8}$ Con el fin de tener información completa y comparable sobre los ingresos de la región, se utiliza la información proveniente de las encuestas de hogares de los países, corregida y ajustada por la División de Estadísticas y Proyecciones Económicas de la CEPAL.
}

proporciones que la brecha favorable para los hombres de los demás países en estudio; mientras que, El Salvador muestra diferencias cercanas a cero, excepto para el último quintil, el cual presenta una brecha a favor de los hombres en 3.1 puntos porcentuales.

De manera complementaria a las brechas de ingreso, se encontró que, según la actividad, las personas ocupadas y las que están estudiando son quienes muestran mejores índices de uso de Internet. Adicionalmente, las mayores brechas de género se presentan entre las y los ocupados: en Chile la brecha es a favor de los hombres en 5 puntos porcentuales y en México de 4.7 puntos. La brecha a favor de las mujeres es para las que dijeron asistir a establecimientos educativos como actividad principal, esto para los casos de Panamá, Brasil y México, sin embargo, 
esta diferencia solo alcanza a ser de 1.5 puntos porcentuales en el mejor de los casos (Panamá).

En general, de los determinantes de uso de Internet que se han visto en esta sección, el nivel de ingreso es quizás el que tiene implicaciones más fuertes en términos de difusión de las TIC en la región, teniendo en cuenta que ha sido validado en la literatura de la región como determinante importante de uso de TIC y a su vez es el determinante principal del acceso a las tecnologías. De acuerdo a la disponibilidad de información de micro datos de la región y habiendo visto que las brechas de género más sobresalientes se presentan en Chile y México, a continuación se presenta la aproximación econométrica utilizada para evaluar el efecto de género, y su interacción con el ingreso, en el uso de Internet en estos dos países de alta difusión tecnológica.

\section{Evidencia Empírica}

Este ejercicio busca básicamente encontrar si, el efecto (positivo) del ingreso sobre la probabilidad de usar Internet, depende del sexo del usuario, es decir, si céteris páribus, los demás determinantes de uso de Internet, y bajo iguales niveles de ingreso, los hombres y las mujeres tienen las misma probabilidad de uso de la tecnología (equidad), o si, por el contrario, céteris páribus, hay diferencias en la probabilidad de uso solamente a razón del sexo de la persona (inequidad digital de género).

De esta manera, lo que se busca calcular es el efecto de género en la posibilidad de usar Internet, para esto se implementó un modelo probabilístico de interacción que combina los principales determinantes de uso de Internet (educación, edad, zona geográfica y condición de ocupación), con la interacción de otros dos determinantes: el sexo de la persona y el ingreso per cápita del hogar?.

A continuación se hace una breve descripción de la construcción de los modelos probabilísticos utilizados.

Desde una aproximación microeconómica del problema, los individuos se enfrentan a la decisión de usar o no Internet sujetos a sus preferencias y restricciones de tiempo e ingreso. Este comportamiento se modela bajo una función de utilidad lineal en donde ambas, la utilidad de usar y la

\footnotetext{
${ }^{9}$ El ingreso representa el logaritmo natural del ingreso per cápita equivalente del hogar de cada individuo.
}

utilidad de no usar Internet para el individuo, se aproximan a una función lineal del vector de características (conjunto de características socioeconómicas del individuo) y de un término de error (término estocástico que recoge las características no observables del individuo).

$U_{i, S}=\alpha_{S}+X_{i} \beta_{S}+\varepsilon_{i, S}$

$U_{i, N}=\alpha_{N}+X_{i} \beta_{N}+\varepsilon_{i, N}$

Donde, $U_{i, S}$ es la utilidad derivada de usar Internet y $U_{i, N}$ es la utilidad derivada de no usar Internet, $X$ es el vector de características socioeconómicas del individuo, y $\varepsilon_{i, S}$ y $\varepsilon_{i, N}$ son los términos de error.

De acuerdo con lo anterior, el individuo $i$ decidirá usar Internet si la utilidad que le genera hacerlo $\left(U_{i, S}\right)$ es mayor que la utilidad que le generará no hacerlo $\left(U_{i, N}\right)$, en otras palabras la probabilidad de que el individuo $i$ use $\circ$ no Internet depende de cuál de las dos utilidades es mayor. Este comportamiento se puede modelar como sigue,

$\operatorname{Prob}\left(U_{s o}=I\right)=\operatorname{Prob}\left(U_{i, S}>U_{i, N}\right)=F\left[\left(\alpha_{S}-\alpha_{N}\right)+X_{i}\left(\beta_{S}-\beta_{N}\right)\right]$

$\operatorname{Prob}($ Uso=I $=\Phi(\theta \mathrm{X})$

Donde, $F$ es la función de distribución acumulativa del término de error, el cual se asume que se distribuye normalmente y por lo tanto el modelo puede ser estimado bajo la metodología de máxima verosimilitud mediante un modelo probabilístico.

El modelo utilizado es un modelo Probit tradicional, pero con la variación de la inclusión de términos de interacción. Los modelos de interacción son ampliamente utilizados en modelos lineales, debido a su alta pertinencia como herramienta de investigación para la aproximación al efecto de una variable sobre otra. Sin embargo, para los modelos no lineales existen algunos problemas de utilización de esta metodología, la mayoría de ellos por imprecisiones de medición $^{10}$.

\footnotetext{
${ }^{10}$ De hecho, Norton et. al. (2004) reportaron que de una revisión de 13 importantes revistas económicas, de los 72 artículos publicados entre 1980 y 2000 , que utilizan términos de interacción en modelos no lineales, solamente uno de ellos calcula e interpreta de manera estrictamente correcta el coeficiente del término de interacción.
} 
El ejercicio empírico aplicado para modelar la probabilidad de uso de Internet de los individuos fue:

$\operatorname{Prob}($ Uso $=I)=\Phi\left(\beta_{1} \cdot\right.$ Ingreso $+\beta_{2} \cdot$ Sexo $+\beta_{12} \cdot$ Ingreso $*$ Sexo + $\beta_{3} \cdot$ Edad $+\beta_{4} \cdot$ Educación $+\beta_{5} \cdot$ Zona $+\beta_{6} \cdot$ Ocupado) (v)
Donde, $\beta_{12}$ es el coeficiente del término de interacción, que es el término de interés para evaluar los efectos marginales de la interacción entre sexo e ingreso. La tabla I define detalladamente las variables implementadas en el modelo.

\begin{tabular}{|c|c|c|}
\hline Variable & Tipo & Definición \\
\hline $\begin{array}{l}\text { Uso de } \\
\text { Internet }\end{array}$ & Dummy & $\begin{array}{l}\text { I- Persona que usa Internet } \\
\text { (Definición para los países en estudio del Partnership } \\
\text { para la medición de las TIC para el Desarrollo) }\end{array}$ \\
\hline Ingreso & Continua & Ingreso per cápita del hogar corregido \\
\hline Sexo & Dummy & I - Mujer \\
\hline Sexo* Ingreso & Dummy * Continua & Variable interactuada \\
\hline Educación & Continua & Número de años de educación de la persona \\
\hline Edad & Continua & Edad de la persona (corregido) \\
\hline Zona & Dummy & I - Zonas urbanas \\
\hline Ocupado & Dummy & I - Persona definida como ocupada \\
\hline
\end{tabular}

Tabla I. Variables del modelo

Ahora bien, la idea conceptual detrás del modelo se basa en el análisis de los coeficientes de las variables y su efecto marginal. Entones, si el coeficiente del término interactuado resulta estadísticamente significativo, y además su magnitud nos deja ver las diferencias por sexo, entonces se estaría probando que existe un factor que es netamente de género que estaría afectando las diferencias de uso de Internet encontradas para algunos países de la región.

Debido a que se está haciendo un análisis para el ingreso per cápita del hogar como el factor determinante más significativo tanto para el acceso como para el uso de Internet, la si significancia de la interacción de género implica que ese efecto positivo del ingreso es diferente para hombres y mujeres. Pero, iqué tan diferente es y a favor de quién?
Para esto, se utilizó una corrección en la metodología desarrollada por Norton et. al. (2004) que permite calcular de manera apropiada la significancia del término interactuado, los errores estándar y los efectos marginales, ya sea para variables interactuadas continuas o discretas. En este caso, la variable interactuada es el producto de una variable dummy (sexo) con una continua (logaritmo natural del ingreso per cápita del hogar).

Adicionalmente, la literatura sugiere que en modelos no lineales, la interpretación de los términos de interacción debe contemplar también el análisis gráfico del comportamiento del efecto marginal. Esto debido a que la estimación derivada del algoritmo no alcanza a captar el verdadero efecto del cambio entre variables interactuadas que si puede ser apreciado mediante el análisis gráfico. La explicación es algebraica y se puede ver en la derivada cruzada para el cálculo del efecto marginal:

$$
\frac{\partial^{2} \Phi(u)}{\partial x_{1} \partial x_{2}}=\beta_{12} \Phi^{\prime}(u)+\left(\beta_{1}+\beta_{12} x_{2}\right)\left(\beta_{2}+\beta_{12} x_{1}\right) \Phi^{\prime \prime}(u)
$$


Como se puede ver en (vi), aunque el coeficiente del término de interacción $\beta_{12}$ fuera igual a cero, es decir, en el caso de que no existiera un efecto interacción debido al sexo de la persona, la derivada cruzada no necesariamente es igual a cero. En este caso el análisis se debe completar con la lectura de las gráficas que se derivan de la metodología Inteff que se aplica en este ejercicio siguiendo a Norton et al. (2004), para evaluar si hay algún efecto interactuado en la probabilidad de uso de Internet.

Por otro lado, a diferencia de los métodos tradicionales de cálculo de efectos marginales en modelos no lineales, el resultado calculado es una distribución de magnitudes del efecto marginal de la variable interactuada. Esto implica cálculos diferenciados para interacciones de variables dummy con continuas, ambas dummy o ambas continuas.

Habiendo dicho esto, la siguiente sección presenta los resultados de los modelos Probit tradicionales y los resultados de la aplicación de la metodología Inteff para el cálculo correcto de los efectos marginales y los errores estándar del modelo, con el fin de contestar a la pregunta principal: ¿el efecto (positivo) del ingreso sobre la probabilidad de usar Internet, depende del sexo del individuo?

\section{Resultados}

La tabla 2 muestra las estimaciones de los modelos Probit de uso de Internet para Chile 2006 y México 2007. Como se esperaba, los coeficientes del ingreso, la zona (urbana) y la educación son positivos para ambos países, mientras que los coeficientes del sexo (mujer), y la edad resultaron negativos tanto para Chile como para México. Adicionalmente, todas las variables mostraron buena significancia.

Las tablas 3 y 4 muestran los resultados de la estimación Inteff para ambos países.

\begin{tabular}{|c|c|c|c|c|}
\hline & \multicolumn{2}{|c|}{ Chile 2006} & \multicolumn{2}{|c|}{ México 2007} \\
\hline Variable & Coeficiente & Significancia & Coeficiente & Significancia \\
\hline Ingreso & $\begin{array}{c}+ \\
1.12 \mathrm{e}-06\end{array}$ & $\begin{array}{c}* * * \\
(0.000)\end{array}$ & $\begin{array}{c}+ \\
.0000977\end{array}$ & $\begin{array}{c}* * * \\
(0.000)\end{array}$ \\
\hline Sexo & $\begin{array}{c}- \\
-.030287\end{array}$ & $\begin{array}{c}* * * \\
(0.000)\end{array}$ & $\begin{array}{c}- \\
-.1018885\end{array}$ & $\begin{array}{c}* * * \\
(0.002)\end{array}$ \\
\hline Interaction & $\begin{array}{c}- \\
-4.93 e-08\end{array}$ & $\begin{array}{c}* * \\
(0.029)\end{array}$ & $\begin{array}{c}- \\
-.0000444\end{array}$ & $\begin{array}{c}* * * \\
(0.000)\end{array}$ \\
\hline Edad & $\begin{array}{c}- \\
-.0295918\end{array}$ & $\begin{array}{c}\text { **** } \\
(0.000)\end{array}$ & $\begin{array}{c}- \\
-.051162\end{array}$ & $\begin{array}{c}* * * \\
(0.000)\end{array}$ \\
\hline Educación & $\begin{array}{c}+ \\
.0286568\end{array}$ & $\begin{array}{c}* * * \\
(0.000)\end{array}$ & $\begin{array}{c}+ \\
.2055487\end{array}$ & $\begin{array}{c}* * * \\
(0.000)\end{array}$ \\
\hline Zona & $\begin{array}{c}+ \\
.5387785\end{array}$ & 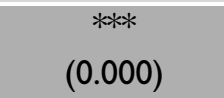 & $\begin{array}{c}+ \\
.4489098\end{array}$ & $\begin{array}{c}\text { **** } \\
(0.000)\end{array}$ \\
\hline Número Obs. & 268.546 & & 17.445 & \\
\hline
\end{tabular}

Tabla 2. Resultados estimación Probit 


\begin{tabular}{|c|c|c|c|c|c|}
\hline México & Obs & Mean & Std. Dev. & Min & Max \\
\hline _probit_phat & 17445 & .2274787 & .2450245 & $8.91 \mathrm{e}-1 \mathrm{I}$ & I \\
\hline _probit_li r & 17445 & $-8.88 e-06$ & $6.36 e-06$ & -.0000177 & 0 \\
\hline probit_ie & 17445 & $-9.88 e-06$ & $6.83 e-06$ & -.0000258 & .0000149 \\
\hline _probit_se & 17445 & $1.69 e-06$ & $1.19 \mathrm{e}-06$ & $1.07 e-18$ & $4.24 e-06$ \\
\hline probit_z & 17445 & $-5.74623 \mid$ & 1.788502 & -8.295686 & 10.46775 \\
\hline
\end{tabular}

Tabla 3. Resultados estimación Inteff para México

\begin{tabular}{|c|r|r|r|r|r|}
\hline Chile & \multicolumn{1}{|c|}{ Obs } & \multicolumn{1}{c|}{ Mean } & Std. Dev. & \multicolumn{1}{|c|}{ Min } & \multicolumn{1}{c|}{ Max } \\
\hline _probit_phat & 268546 & .2473484 & .1841825 & .0001468 & 1 \\
\hline _probit_li r & 268546 & $-1.28 \mathrm{e}-08$ & $6.27 \mathrm{e}-09$ & $-1.97 \mathrm{e}-08$ & 0 \\
\hline _probit_ie & 268546 & $-1.8 \mathrm{Ie}-08$ & $7.10 \mathrm{e}-09$ & $-4.46 \mathrm{e}-08$ & $4.66 \mathrm{e}-08$ \\
\hline _probit_se & 268546 & $5.85 \mathrm{e}-09$ & $2.85 \mathrm{e}-09$ & 0 & $1.66 \mathrm{e}-08$ \\
\hline _probit_z & 268542 & -3.551896 & 1.276807 & -6.225398 & 6.60591 \\
\hline
\end{tabular}

Fuente: Cálculos propios con base en Sistema de Información OSILAC

Tabla 4. Resultados estimación Inteff para Chile

De acuerdo a las tablas 2 y 3 , tanto para Chile como para México, el efecto marginal del término de interacción resultó negativo, además de significativo. Adicionalmente, los resultados Inteff permiten calcular la magnitud correcta de este efecto marginal. Esto se puede ver de manera más clara con el análisis gráfico.

Los gráficos 7 y 8 confirman la significancia del coeficiente del término de Interacción mostrando como existe un efecto en el hecho de ser mujer con respecto a la probabilidad de usar Internet (eje $\mathbf{x}$ de los gráficos). También se puede ver la diferencia entre la estimación de este efecto, hecha por el método tradicional (lineal en los gráficos) y la estimación, distribución de efectos, hecha por la metodología aplicada (nube de puntos en los gráficos).

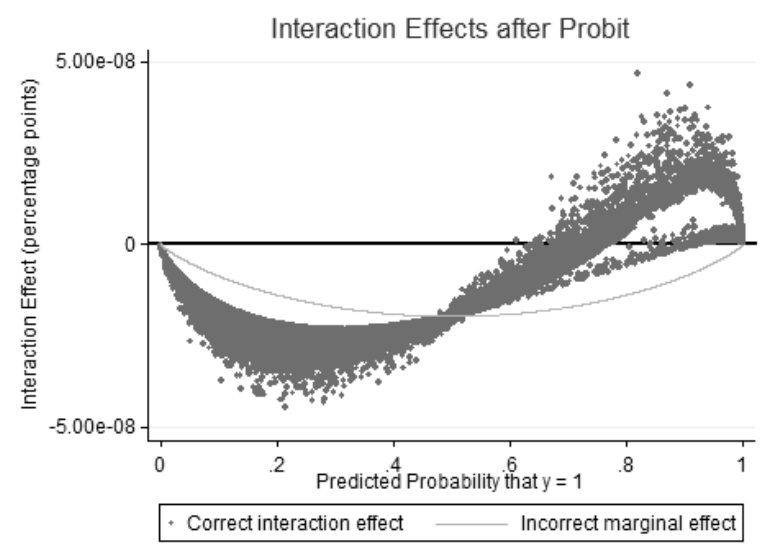

Gráfico 7. Resultados gráficos estimación Inteff para Chile.

Fuente: Cálculos propios con base en Sistema de Información OSILAC 


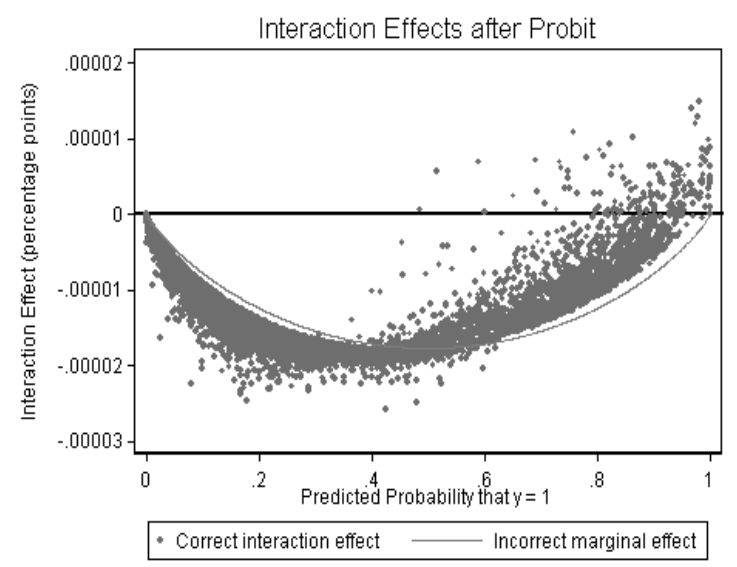

Gráfico 8. Resultados gráficos estimación Inteff para México.

Fuente: Cálculos propios con base en Sistema de Información OSILAC

\section{Conclusiones}

La primera reflexión es que los micro datos permitieron mostrar evidencia del uso diferenciado de Internet entre mujeres y hombres, el cual se presenta de forma más marcada en países con alta difusión de TIC como Chile, Brasil y México. De esta manera se pudo observar que las diferencias de género prevalecen a lo largo de diferentes edades, niveles educativos y niveles de ingreso.

Adicionalmente se mostró cómo los indicadores de uso de Internet, son mejores para las mujeres en países con bajos índices de digitalización (i.e., Panamá), y no es así en países que han alcanzado altos niveles de acceso y uso a las TIC (i.e., México, Brasil y Chile), en donde la condición de feminidad tiene un efecto negativo en la probabilidad de usar Internet. Los hombres, jóvenes, habitantes urbanos y personas más ricas y educadas tienen mayor probabilidad de usar Internet.

La metodología utilizada en el ejercicio econométrico brinda, en efecto, una mejor aproximación al cálculo de los efectos marginales de cada uno de los determinantes, siendo mayor la diferencia entre el cálculo tradicional y el cálculo corregido en las estimaciones hechas para Chile.

Los resultados derivados de la aplicación de la estimación Inteff con Chile y México, muestran que evidentemente hay diferencias en el efecto del ingreso como determinante de uso de Internet, que depende del sexo de la persona. Este efecto es negativo para las mujeres en la interacción con el ingreso. Esto quiere decir que el cambio positivo en el ingreso tiene un menor efecto en la probabilidad de usar Internet para las mujeres. En el caso de Chile, para algunos individuos con una probabilidad estimada de usar Internet mayor a 0.7 el efecto de la variable interactuada es positivo (Gráfico 7). Para el caso de México, este efecto es negativo para casi todos los individuos (Gráfico 8).

De esta manera se puede afirmar que, en correspondencia con la literatura, también para América Latina existe evidencia de un patrón diferenciado de difusión de Internet en la región, en particular hay señales de patrones de difusión de la tecnología diferentes para mujeres y hombres. Aunque no se identifica un patrón similar para todos los países, se encuentran algunas tendencias compartidas, en este caso, entre países con alta difusión de la tecnología como Chile y México. Al analizar estadística y econométricamente las diferencias de género para estos dos países, se logró legitimar la noción de un diferencial de uso entre mujeres y hombres de la región que trasciende parámetros como ubicación geográfica, edad, niveles de ingreso y de educación. En otras palabras, para Chile y México se encuentra una implicación de género en el uso de Internet que requiere ser abordada por su importancia para políticas públicas nacionales de TIC y de equidad. En el mismo sentido se considera pertinente que en la medida de las posibilidades y de la disponibilidad de los datos, se amplíe el estudio para analizar y contrastar estos resultados con información de otros países de la región.

ISSN: 07I 8-2724. (http://www.jotmi.org)

Journal of Technology Management \& Innovation (C) Universidad Alberto Hurtado, Facultad de Economía y Negocios 


\section{Consideraciones Finales y de Política Pública}

Así como la región ha avanzado en el acceso a las TIC y más aún en crear una sociedad de la información incluyente y que promueva el alfabetismo digital, todavía sobresalen algunas brechas que deben empezar a ser trabajadas. En este sentido, cabe resaltar algunas buenas prácticas que pueden ser tenidas como referencia para una mayor inclusión de la mujer en la Sociedad de la Información.

Existen algunas iniciativas que han logrado cerrar brechas desde acciones positivas de política pública. El Women's Technology Empowerment Center W.TEC de Nigeria es un ejemplo de estas buenas prácticas. Creado con el fin de ser una librería digital para equidad de género en ciencia y lenguaje, este centro ha permitido que, a través del acceso y uso de tecnologías como Internet, las mujeres de la región tengan un mayor alfabetismo digital y puedan aspirar a tener cargos importantes que han estado tradicionalmente asignados a personas que han salido del país a entrenarse. Adicionalmente se ha visto el impacto positivo de este tipo de prácticas en la independencia financiera de las mujeres. (GLOBAL ALLIANCE FOR ICT AND DEVELOPMENT GAID, 2009).

Por otro lado, un caso que se destaca en América Latina es el Centro Latinoamericano y del Caribe en Género y Sociedad de la Información que fue impulsado por los desarrollos de la UNESCO con su cátedra regional sobre Mujer, Ciencia y Tecnología en América Latina. Este centro es una de las principales alianzas e iniciativas de investigación sobre el rol de las mujeres en la Sociedad de la Información, promoviendo el uso de las TIC para el logro y mejora de los procesos de educación, productividad, cultura e innovación de las mujeres de la región".

Adicionalmente, la región ha hecho importantes esfuerzos en la medición del impacto de las TIC en la vida de las mujeres. La metodología GEM (Gender Evaluation Methodology) es uno de esos esfuerzos en medición cuyo objetivo principal es brindar herramientas para la evaluación de proyectos de TIC con perspectiva de género. El informe regional para América Latina dejó ver como algunos proyectos de TIC implementados en

\footnotetext{
" http://www.catunescomujer.org/cgysi-lac/presentacion.html\#
}

México, Colombia, Ecuador, Bolivia y Brasil $^{12}$, se encontraron con mujeres que han sentido poca confianza $y$ discriminación en el uso de las TIC como consecuencia del proceso cultural que ha atribuido los roles tecnológicos a los hombres (ASSOCIATION FOR PROGRESSIVE COMMUNICATIONS, 2003). Sin embargo, el informe también da cuenta de que cada política pública orientada a la promoción del acceso o el uso de las TIC entre las mujeres, tiene un impacto netamente positivo no solamente en términos de inclusión digital, sino también en términos de potenciales herramientas de inclusión social y económica para las mujeres.

La recomendación principal en este sentido es que las acciones a favor de la inclusión de las mujeres en la Sociedad de la Información en América Latina, prevean los mecanismos de evaluación desde el momento mismo de la planeación de las políticas, teniendo en cuenta que los impactos pueden ser tanto a nivel de inclusión digital (aumento del acceso y uso de las TIC), como a nivel de inserción social y económica (impactos positivos en educación, salud, generación de ingresos, empleo, calidad de vida, etc.).

La mayoría de las acciones positivas exitosas y buenas prácticas de inclusión digital, especialmente de equidad de género, se han sustentado en políticas de largo plazo que cuentan con la solidez presupuestal para ser implementadas. Así, las políticas públicas en materia de TIC de la región tienen que planear presupuestos sensibles al género que respalden iniciativas de reducción de brechas de género no solamente en el acceso y el uso, sino también en los contenidos, desarrollos y decisiones que se pueden tomar a través de las tecnologías.

El caso de las TIC y especialmente de Internet como mecanismo de inserción económica y de generación de ingresos y oportunidades para las mujeres, las iniciativas deben venir acompañadas de un respaldo presupuestal que garantice la viabilidad y sostenibilidad de las políticas, con el fin de evitar una posterior mayor marginalidad de las

\footnotetext{
12 Algunos otros países están buscando capacitarse en la implementación de la herramienta GEM para la evaluación de sus políticas de TIC y género, tal es el caso de República Dominicana, que a través de su Instituto de las Telecomunicaciones INDOTEL, ha venido promoviendo la capacitación a nivel internacional con la participación de México, El Salvador, Costa Rica, Panamá y Guatemala.
}

ISSN: 07I 8-2724. (http://www.jotmi.org)

Journal of Technology Management \& Innovation () Universidad Alberto Hurtado, Facultad de Economía y Negocios 
mujeres y brindar un efectivo empoderamiento de ellas en la Sociedad de la Información.

Aunque las acciones positivas o más aún las políticas públicas para las mujeres en la Sociedad de la Información no puedan resolver todos los problemas, en especial aquellos básicos, relacionados con los Objetivos de Desarrollo del Milenio, las TIC si son una herramienta que se constituye en una vía por la cual las mujeres pueden tener puntos de encuentro, alternativas y soluciones a los diferentes problemas que se encuentran día a día.

Para la superación de la pobreza y para el desarrollo de los países de la región en general, el objetivo de investigación no son tanto las TIC como el uso de las mismas. Es en el uso de esas herramientas en donde los individuos, las comunidades y la sociedad de los países en desarrollo pueden alcanzar resultados positivos y oportunidades a través del uso de las tecnologías. Sobran los ejemplos de emprendimientos surgidos a través del uso del Internet o incluso de los teléfonos móviles donde a la distancia de un click se crea la oportunidad de capacitación, de instalar un negocio o de la generación de ingresos. Esto por ejemplo minimiza el riesgo de vulnerabilidad, por ende favorece los procesos de superación de la pobreza y el desarrollo.

Sin embargo, es preciso tener en cuenta que el éxito de los procesos de TIC en la Sociedad de la Información y su impacto positivo para el desarrollo, en particular para las mujeres, va a depender también de los roles de género que les sean asignados en cada cultura, en cada sociedad, y un primer paso para vencer esas barreras es el reconocimiento de las diferencias y de las brechas que aún persisten, así como la búsqueda por mantener cerradas las brechas que ya se han superado

\section{Acknowledgment}

Este documento fue producido en el marco del Programa Sociedad de la Información de la División de Desarrollo Productivo y Empresarial de la CEPAL. Las opiniones expresadas en este documento son de exclusiva responsabilidad de la autora y pueden no coincidir con las de la organización. La autora agradece los comentarios de la División al documento y los valiosos aportes de Jacopo Gamba y Matteo Grazzi.

This document was prepared within the research activities of the Information Society Programme of the Division of
Production, Productivity and Management of UN-ECLAC. The opinions presented in this paper are full responsibility of the author and might not coincide with those of the organization. The author thanks the Division for the comments to the document, as well as the remarkable contributions of Jacopo Gamba and Matteo Grazzi.

\section{Referencias}

ASSOCIATION FOR PROGRESSIVE COMMUNICATIONS (2002). Gender Evaluation Methodology for ICT Initiatives. Interim Report.

BIMBER, B. (2000). Measuring the Gender Gap on the Internet. University of California, Santa Barbara.

CASTAÑO, C. (2005). Las mujeres y las tecnologías de la información. Internet y la trama de nuestra vida. Instituto Andaluz de la Mujer, Madrid.

CENTRO LATINOAMERICANO Y DEL CARIBE EN GÉNERO Y SOCIEDAD DE LA INFORMACIÓN. http://www.catunescomujer.org/cgysi-lac/presentacion.html

CHEN, D. (2004). Gender Equality and Economic Development: The Role for Information and Communication Technologies. The Knowledge for Development Program. The World Bank. Washington DC.

CRISTANCHO C., Sánchez M.. (2009). Compendio de Prácticas sobre Implementación de Preguntas de TIC en Encuestas de Hogares y Empresas Revisión marzo 2009. Mimeo, documento preliminar del Observatorio de la Sociedad de la Información para Latinoamérica y el Caribe, de la División de Desarrollo Productivo y Empresarial. CEPAL.

GLOBAL ALLIANCE FOR ICT AND DEVELOPMENT, GAID (2009). A Digital Shift: Youth and ICT for Development. Best Practices. New York.

GRAZZI, M., Vergara, S. (2008), What Drives ICT Diffusion in Developing Countries? Evidence from Paraguay. Mimeo. Documento del Observatorio de la Sociedad de la Información para Latinoamérica y el Caribe, de la División de Desarrollo Productivo y Empresarial. CEPAL.

GRAZZI, M. (2009), Patterns of Internet Use in Latin America. Mimeo. Documento del Observatorio de la Sociedad de la 
Información para Latinoamérica y el Caribe, de la División de Desarrollo Productivo y Empresarial. CEPAL.

HARGITTAI, E., Shafer, S. (2006). Differences in Actual and Perceived. Online Skills: The Role of Gender. Social Science Quarterly, 87(2), 432-448.

KENNEDY, T., Wellman, B., Klement, K. (2003). Gendering the Digital Divide. It \& Society, I(5), summer 2003, 72-96.

NORTON, E., Wang, H., Ai, C. (2004). Computing interaction effects and standard errors in logit and probit models. The Stata Journal, 4(2), 154-167.

ORGANISATION FOR ECONOMIC CO-OPERATION AND DEVELOPMENT, OECD (2007). Working Party on the Information Economy. ICTs and Gender. Paris

----- (2009). Guide to Measuring the Information Society. Paris.

ONO, H., Zavodny, M. (2003). Gender and the Internet. Social Science Quarterly, 84, III-12 I

PARTNERSHIP PARA LA MEDICIÓN DE LAS TIC PARA EL DESARROLLO (2005). Indicadores clave de las tecnologías de la información y de las comunicaciones. Naciones Unidas, Santiago de Chile.

SÁNCHEZ, M. (2009). ¿Brecha digital, brecha de género? Mujeres y Hombres en América Latina: Panorama Regional de Difusión de Computadora e Internet. Mimeo. Documento del Observatorio de la Sociedad de la Información para Latinoamérica y el Caribe, de la División de Desarrollo Productivo y Empresarial. CEPAL.

SHASHAANI, L. (1997). Gender differences in computer attitudes and use among college students. Journal of Educational Computing Research, I6(I), 37-5I.

UNITED NATIONS ECONOMIC COMMISSION FOR EUROPE UNECE (2004). Access to Financing and ITC for Women Entrepreneurs in the UNECE Region. Challenges and good practices. Geneva and New York. 


\section{Anexos}

\section{Encuestas de Hogares Utilizadas, América Latina}

\begin{tabular}{|c|c|c|}
\hline País & Nombre de la encuesta & Tipo de encuesta \\
\hline Brasil & $\begin{array}{l}\text { Pesquisa Suplementar sobre acceso a Internet da } \\
\text { Pesquisa Nacional por Amostra de Domicilios PNAD }\end{array}$ & Encuesta de hogares de propósitos múltiples \\
\hline Chile & $\begin{array}{l}\text { Encuesta de Caracterización Socioeconómica Nacional } \\
\text { CASEN }\end{array}$ & Encuesta de hogares de propósitos múltiples \\
\hline El Salvador & Encuesta de Hogares de Propósitos Múltiples. EHPM & Encuesta de Hogares de Propósitos Múltiples \\
\hline México & $\begin{array}{l}\text { Encuesta Nacional sobre Disponibilidad y Uso de las } \\
\text { Tecnologías de la Información en los Hogares ENDUTIH }\end{array}$ & $\begin{array}{l}\text { Encuesta sobre TIC adjunta a encuesta de } \\
\text { empleo. }\end{array}$ \\
\hline Panamá & Encuesta de propósitos múltiples EPM & Encuesta de hogares de propósitos múltiples \\
\hline República Dominicana & $\begin{array}{l}\text { Encuesta Nacional de Hogares de propósitos múltiples } \\
\text { ENHOGAR }\end{array}$ & Encuesta de hogares de propósitos múltiples \\
\hline Uruguay & $\begin{array}{l}\text { Encuesta Nacional de Hogares Ampliada ENHA } 2006 \text { y } \\
\text { Encuesta Continua de Hogares ECH } 2008\end{array}$ & Encuesta de hogares de propósitos múltiples \\
\hline
\end{tabular}

Fuente: OSILAC, Sistema de Información Estadístico de TIC a junio de 2009

\section{Estimación Probit para México}

\begin{tabular}{|c|c|c|c|c|c|c|}
\hline \multirow{2}{*}{\multicolumn{2}{|c|}{\begin{tabular}{|l|} 
Probit regression \\
LR chi2(7)
\end{tabular}}} & \multicolumn{2}{|c|}{ Number of obs } & \multicolumn{2}{|l|}{$=$} & 17445 \\
\hline & & \multicolumn{2}{|l|}{$=$} & \multicolumn{2}{|c|}{6356.11} & \\
\hline \multicolumn{2}{|c|}{ Prob > chi2 } & \multicolumn{2}{|l|}{$=$} & 0.0000 & & \\
\hline \multicolumn{2}{|c|}{ Log likelihood $=-6200.1037$} & \multicolumn{2}{|c|}{ Pseudo R2 } & \multicolumn{2}{|l|}{$=$} & 0.3389 \\
\hline inter & Coef. & Std. Err. & $z$ & $P>z$ & [95\% Conf. & Interval] \\
\hline ingperhog & .0000977 & $6.96 e-06$ & 14.03 & 0.000 & .000084 & .0001113 \\
\hline sexo & -.1018885 & .0334605 & -3.05 & 0.002 & -.1674698 & -.0363072 \\
\hline otroit & -.0000444 & $8.37 e-06$ & -5.30 & 0.000 & -.0000608 & -.000028 \\
\hline edad & -.051162 & .0014118 & -36.24 & 0.000 & $-.053929 \mid$ & -.0483949 \\
\hline a du & .2055487 & .0039125 & 52.54 & 0.000 & .1978803 & .2132171 \\
\hline zona & .4489098 & .0266616 & 16.84 & 0.000 & .3966541 & .5011655 \\
\hline ocupad & $-.3557 \mid 72$ & .0321849 & -11.05 & 0.000 & -.4187984 & -.292636 \\
\hline cons & -1.422892 & .036653 & -38.82 & 0.000 & $-|.49473|$ & -1.351054 \\
\hline
\end{tabular}

Fuente: Cálculos propios con base en Sistema de Información OSILAC 


\section{Estimación Probit para Chile}

\begin{tabular}{|c|c|c|c|c|c|c|c|}
\hline \multirow{2}{*}{\multicolumn{2}{|c|}{$\begin{array}{l}\text { Probit regression } \\
\text { LR chi2(7) }\end{array}$}} & \multicolumn{2}{|c|}{ Number of obs } & \multicolumn{2}{|l|}{$=$} & \multicolumn{2}{|c|}{268546} \\
\hline & & $=$ & & \multicolumn{2}{|c|}{58247.85} & & \\
\hline \multicolumn{2}{|c|}{ Prob > chi2 } & $=$ & & \multicolumn{2}{|c|}{0.0000} & & \\
\hline \multicolumn{2}{|c|}{ Log likelihood $=-122922.06$} & \multicolumn{2}{|c|}{ Pseudo R2 } & \multicolumn{2}{|l|}{$=$} & \multicolumn{2}{|c|}{0.1915} \\
\hline inter & Coef. & Std. Err. & $z$ & $\mathrm{P}>\mathrm{z}$ & {$[95 \%$} & Conf. & Interval] \\
\hline ingperhog & $1.12 \mathrm{e}-06$ & $1.48 \mathrm{e}-08$ & 76.24 & 0.000 & $1.10 \mathrm{e}$ & -06 & $1.15 \mathrm{e}-06$ \\
\hline sexo & -.030287 & .0068005 & -4.45 & 0.000 & -.043 & 6158 & -.0169582 \\
\hline otroit & $-4.93 e-08$ & $2.26 \mathrm{e}-08$ & -2.18 & 0.029 & -9.36 & $e-08$ & $-4.94 \mathrm{e}-09$ \\
\hline edad & -.0295918 & .0001922 & -153.96 & 0.000 & -.029 & 9686 & $-.0292|5|$ \\
\hline a du & .0286568 & .0003168 & 90.45 & 0.000 & .0280 & 358 & .0292777 \\
\hline zona & .5387785 & .0063536 & 84.80 & 0.000 & .5263 & 257 & .5512314 \\
\hline ocupad & $-.06|33| 4$ & .0071762 & -8.55 & 0.000 & -.075 & 3966 & -.0472662 \\
\hline _cons & -.5266007 & .0073778 & -71.38 & 0.000 & $-.54 \mid$ & 0608 & $-.512 \mid 405$ \\
\hline
\end{tabular}

Fuente: Cálculos propios con base en Sistema de Información OSILAC 